\title{
The Influence of Ancient Chinese Literati on the Artistic Development of Guqin
}

\author{
Guozhi Chen \\ Sichuan University of Arts and Science \\ Dazhou, Sichuan, 635000
}

\begin{abstract}
As the most typical musical instrument of Chinese traditional music, the development of Guqin is deeply influenced by ancient Chinese literati. The participation of literati has endowed Guqin with rich cultural and humanistic significance, formed a unique set of Guqin music aesthetics, and left a rich cultural heritage of Guqin melody, Guqin theory and Guqin works, etc.
\end{abstract}

Keywords-Guqin; literati; credit and contribution; obstruction and harm

\section{INTRODUCTION}

Guqin, also known as "Lyre" or "Yaoqin", received its name in modern times because of the time-honored history. As one of the earliest Chinese plucked instrument, it is regarded as the most representative Chinese traditional music instruments. However, the once glorious instrument that lasted for thousands of years has gradually disappeared in modern times, and even fell into the fate of being listed as "World Intangible Cultural Heritage". The reason surely is related to its own shape and artistic features, as well as the background of the social and cultural development of the Chinese nation. However, the most direct and crucial factor determining the development track and destiny of Guqin art is the participation of ancient Chinese literati. During the thousands of years' feudal society in ancient China, literati were the mainstream of Guqin performers, as well as the audience of Guqin music. Therefore, the literati had a profound influence on the development of Guqin art, and it can even be said that they has determined the development of Guqin.

\section{THE CLOSE RELATIONSHIP BETWEEN LITERATI AND GUQIN}

"Literati" is a special group of Chinese feudal society lasted for a long time which is closely related to the development of the ancient feudal bureaucrat "scholar" class. "Literati" and "scholar" were not the same concept at the early times. Due to the influence of Confucianism's "Officialdom is the natural outlet for good scholars, scholar is the natural outlet for good officials", the "scholar" class has endowed the characteristics of literati. In the Han Dynasty, the understanding toward "scholar" has become unified that "scholar" grew into literati who can "know the past and the present, distinguish the past and the future", and the boundary between literati and "scholar" has been blurred. Both "literati" and "scholar" can be called "literati". In the aspect of culture property, they were the prophets and disseminators of intellectuals and cultures; in the aspect of political property, they shouldered the mission and responsibility of assisting politics and educating people.

It can be seen from ancient records that before the Spring and Autumn Period, Guqin, as an ordinary folk musical instrument, was widely circulated in the folk. For example, eight Guqin songs were collected in the "Book of Songs", the first anthology of poetry. In the "Strategies of the Warring States, Strategies of Qi", there was also description of "its people playing the musical instrument of Yu, Se, Zhu and Qin", which showed that Guqin has very free form of performance, playing occasions and the aesthetic orientation during the early stage. From the Spring and Autumn Period to the Qin and Han Dynasties and the Wei and Jin Dynasties, the relationship between the literati and the Guqin grew closer with the gradual growth of the literati and "scholar". Since Wei and Jin Dynasties, the status of Guqin has been raised by the literati that Guqin was given the role of self-cultivation and ruling the country and bringing the people stability. For example, "The Book of Rites, Rites of Qu" recorded that "a scholar wouldn't abandon Qin and Se without a reason"; "New Theory, Qin Theory" recorded that "among the eight categories of musical instrument in ancient orchestra, Qin is the best"; "Annotation of Literature and Customs" recorded that "Qin is the most often used instrument of a man of noble character that never being left"; Chen Yang in the Song Dynasty said in his "Yue Shu" that "Qin plays the leading role in music". These concepts and ideas were all proposed by the literati and scholars of that times. Such records proved that Guqin has become the ideal tool of self-cultivation and life achievement of the literati, and gradually evolved to be the special musical instrument of literati that excluded the ordinary people. The development of Guqin has been deeply influenced by the literati that they shared the same fate, and Guqin music even became synonymous with "literati music".

\section{THE CREDIT AND CONTRIBUTION OF LITERATI TO THE ARTISTIC DEVELOPMENT OF GUQIN}

\section{A. Profound Humanism Connotation}

Whether from the birth and shape of Guqin, or from the performance style, Guqin has been endowed with many personified symbolic meanings, with rich humanistic significance and cultural connotations. At this point, it can be 
said that none of the instruments in our country or in the world can rival it. However, it can not do without the work of literati.

There were many recordings of the came into being of Guqin in the ancient books. For example, Cai Yong said in "Qin Cao" that "Fuxi has made Qin in the past to achieve selfcultivation". "New Theory, Qin Theory" of Huan Tan recorded that "Shen Nong used Paulownia to made Qin and took rope as the string, and thus he became the divinity". "The Book of Rites, Rites of Qu" recorded that "Shun has made a fivestringed Qin to sing Nanfeng." All these recordings have associated the create of Guqin with the legendary Chinese ancestors Three Emperors and Five Sovereigns such as Fuxi, Shennong, the Yan Emperor, the Yellow Emperor, Yao and Shun, etc., leaving many beautiful legends, which is also legendary, literary and with rich humanistic connotation.

The shape of the Guqin has also been endowed with many symbolic meanings of Chinese culture. Each part of Guqin has a very vivid name, such as Yueshan, Fengshe, Guanjiao, Yaozhong, Chenglu, Longyin, Yanzu, etc., which are related to the head, forehead, neck, shoulder, waist, foot of people, and thus make Guqin personified. The length of Guqin is three Chi six Cun and six Fen, which is considered to represent 366 days of a year; "broad in the front and narrow at the back" symbolize the social hierarchical relationship; "round on the top and square at the bottom" symbolize the differences between heaven and earth; the five strings Gong, Shang, Jiao, Hui, and Yu symbolize the five social classes of Emperor, subjects, civilian, affair, and things; the sixth and seventh strings are called Wen and $\mathrm{Wu}$ that symbolize the cooperation between emperor and his subjects (see "Bai Hu Tong"); there are three tones in performing Guqin, namely overtone, pushed tone and scattered note, which symbolize the harmonious relationship among the heaven, earth and people (See "Qin Cao" of Cai Yong)... Although these symbolic meanings are too much farfetched, they reflect the meaning that "writings are for conveying truth" and "teach people". It can be seen that the literati who are deeply influenced by Confucianism also attribute the shape of Guqin with the meaning of educating moral virtues. The shape of Guqin is no doubt very beautiful, as said by Liu Chenghua, "As a whole, it is like a symmetrical and complete human body, compact, coordinated and of medium size, with human charm." [1]

\section{B. Unique Guqin Aesthetics}

As scholars generally have higher literary accomplishment, they are good at summing up and discussing Guqin in the process of Guqin practice, exploring the aesthetics principle and standard of performing Guqin and expressing their philosophy thoughts and aesthetic values through Guqin music, and thus form a systematic aesthetic theory through write books and set up theories, and regulate the performance and appreciation of Guqin art.

The ancient Chinese literati were deeply influenced by Confucianism and Taoism, which led to the distinctive tendency of Confucianism and Taoism in Guqin aesthetics. Especially that the Confucian culture has the most profound influence on Guqin aesthetics, and it always occupies the mainstream of Guqin aesthetics. The Qin theory from Qin
Dynasty to Han dynasty laid the foundation for the aesthetic thought of Confucianism Guqin. For example, "Zuo Zhuan" mentioned that "a gentleman playing Qin and Se is for selfcultivation rather than sapping his spirit in seeking pleasures"; "Bai Hu Tong" proposed that "Qin is to forbid dishonest practices and correct human mind". The concept that music should be rooted in $\mathrm{Li}$ and serve for politics has affected the aesthetic development of Guqin for almost 2000 years. "Forbidden" here refers that there are many prohibitions, regulations and restrictions in performing Guqin, no random and free performance, and thus people propose the aesthetic view of neutralization and peace and the musical aesthetic requirements of "joyous but not indecent, mournful but not distressing", which still influenced the performing style of Guqin until now.

Taoism, originated from the Spring and Autumn Period and represented by $\mathrm{Lao} \mathrm{Zi}$ and $\mathrm{Zhuang} \mathrm{Zi}$, also has profound influence on Guqin aesthetics. "Lao Zi" proposed that "the great sound seems soundless" and advocated the music style of "simple and plain"; Zhuang Zi advocated nature-orientation and proposed the aesthetic concept that "playing Qin is enough for self-entertainment" (Zhuangzi, Rang Wang). The thoughts of Lao $\mathrm{Zi}$ and Zhuang $\mathrm{Zi}$ were absorbed and developed by the writers and artists of later generations, such as Ruan Ji, Ji Kang, Bai Juyi, Zhou Dunyi and Xu Shangying, etc., and formed the music aesthetics of simple and peace orientation, which became the aesthetic standard advocated by both Confucianism and Taoism. The major representations are the pursuits of the implicit beauty, profound artistic conception and the implication of the music, and the requirements to the player of disregarding utility and oneself and pursuing harmony between man and nature and the free aesthetic realm integrated with nature.

\section{Many Qin Music, Theory and Works Were Left}

During the more than two thousand years' history from Qin Dynasty to the late Qing Dynasty, the literati played, discussed, and created Qin music and wrote books on Qin, and left over 3000 Qin music and more than 100 works on Qin theory, which became the rich cultural heritage of China. Therefore, the literati had made a name in history. For example, "Yang Chun Bai Xue" and "Nie Zheng Ci Han Wang" in pre-Qin Period, "Guang Ling San", "Jiu Kuang" and "Mei Hua San Nong" in the Wei and Jin Periods, "You Lan" and "Wu Ye Ti" in the Northern and Southern Dynasties, etc. There were even more Qin music after the Sui and Tang Dynasties. Comparatively, Qin throry in Song and Yuan Dynasties were richer, such as Zhu Changwen's "Qin History" and Cheng Yujian's "On Qin” and so on. Since the Ming Dynasty, several different schools have been formed because of the differences on playing style and aesthetic pursuit, and the Qin music and works were also left respectively. In addition, there were still many famous literati in different dynasties who not only engaged in Qin, but also expressed their views on Guqin through poem, calligraphy and painting, etc. These works have added special charm to Guqin art, such as "Qin Tea" of Bai Juyi, "Gui Qu Lai Ci” of Tao Yuanming, "Zui Wong Yin” of Su Shi, and "Gu Yuan" of Jiang Baishi, etc. ${ }^{[2]}$ 
All in all, it was because of the participation of the literati that a large amount of Guqin works can be left and Guqin music stood out in traditional Chinese music, "the perfect notation, rich works, systematic playing theory and aesthetics concept were the contributions of literati fundamentally., ${ }^{[3]}$ It was because of this that Mr. Huang Xiangpeng regarded that, "the significance of Guqin music to Chinese music history is similar to the significance of piano to the history of European music. ${ }^{[4]}$

\section{THE OBSTRUCTION AND HARM OF LITERATI TO THE DEVELOPMENT OF GUQIN ART}

\section{A. Value Theory Rather Than Art, Obstructing the Diversified Development of Guqin}

The ancient Chinese literati were greatly influenced by Confucianism, which regarded educating people as the primary function of music. As a result, the education function of Guqin was over emphasized since the Han Dynasty, but the performance techniques and methods were restricted, demanding the "natural and peaceful" Qin music and against the free expression of emotion. During the Song and Yuan Dynasties, Guqin aesthetics even went to extremes. For example, literati like Fan Zhongyan and $\mathrm{Zhu} \mathrm{Xi}$ and so on denied the artistic function and aesthetic value of Guqin fundamentally, and were against folk music, pleasant music and sad music, which were believed as "the music presaging the fallen state". During the Ming and Qing Dynasties, the artistic quality and creativity of Guqin were totally denied, and many taboos were put forward. Guqin became a tool completely and lost its artistic significance.

Although Taoism opposed Confucianism's concept of taking Guqin as a education tool, the aesthetics of Lao Zi's "great sound seems soundless" and Zhuang Zi's music style of "simple and plain" have deep influence on the later literati that they advocated simple, peaceful and few voices and selfentertainment, taking Guqin as a tool of health maintenance and self-entertainment but ignoring its artistic quality. Even there were a few Taoist scholars, such as Ji Kang and Li Zhi, affirmed the artistic quality and advocated the free development of Guqin art, they still failed to be the mainstream and occupied the main position of Guqin aesthetics thought and practice.

It can be seen that both Confucianism and Taoism take self-cultivation and health maintenance as the artistic function of Guqin. Literati neither pursued the complexity and variety of musical forms and performing techniques, nor pursued the volume expansion or technical content of musical instruments. They belittled folk music and ethnic music and excluded canto and sad sound, which limited the free and diversified development of Guqin to a large extent.

\section{B. Exclude Folk and Advocate Elegant, Separate from the Public, Pushing the Decline of Guqin}

After the Eastern Han Dynasty and the Wei and Jin Dynasties, Guqin became a special instrument for the literati, and the aesthetic orientation of the literati inevitably affected the aesthetic standards and aesthetic style of Guqin. Literati of both Confucianism and Taoism regarded simple and peaceful as the aesthetic standard and took Guqin as a tool of selfcultivation and health maintenance, but ignored the artistic quality. Therefore, literati all exclude the changeable music and various folk music, opposed canto and sad sound, and tried to make Guqin more and more elegant and peaceful. Literati and Qin player of different dynasties all took excluding folk, advocating elegant and rectifying Qin style as their aim that they have made requirements and restrictions on the player, audience and playing content of Guqin.

Since the Song and Yuan Dynasties, the confine of Guqin aesthetics has been further expanded. The status of Guqin was highly sanctified. The restrictions on the player and performance occasion have gone to the extreme. For example, in "Xi Shan Qin Kuang" of Xu Shangyin in Ming Dynasty, it was stated that "Qin was not allow to be in singing and dancing area, and cannot be accompanied by other stringed and woodwind instruments"; in "Qin Pu He Bi Da Quan", Yang Biao has stated 14 occasions that prohibited playing Qin, including "thunder and rain, solar and lunar eclipse, to folk people, to businessman, to whore", etc.

Such high requirements definitely kept the common people away, and thus Guqin can only be played and appreciated in the upper classes or among the literati. On the other hand, ordinary people were away from Guqin due to the even more elegant and peaceful music that didn't entertain their aeshetics. At the end of Qing Dynasty, the great changes of social system led to the decline of literati class, "With the skin gone, to what can the hair attach itself", Guqin eventually declined because of the loss of supporting object and the existence value.

\section{CONCLUSION}

As mentioned above, the literati have forged an indissoluble bond with the Guqin and influenced the development of Guqin deeply. Nowadays, the artistic value and humanistic value of Guqin have been rediscovered and valued by people. Guqin no longer only belong to the literati, more and more people engaged in playing, teaching and learning Guqin. However, there is still a long way for the future of Guqin, and it needs research, exploration and discussion on how to develop Guqin. The author believes that the urgent task at present is to discard the traditional aesthetics of Guqin, build up and popularize the modern aesthetic concept which not only in line with the aesthetic requirements of modern people, but also without losing its inherent aesthetic taste. In addition, more efforts also need to be done in creating new music and performance techniques, so as to make Guqin popular in the public, return art to Guqin and give Guqin to people. Only in this way can make Guqin, a special art form that can best represent the characteristics of Chinese traditional music, be reborn and glorious again.

\section{REFERENCES}

[1] Liu Chenghua. The Beauty of Molding and Decoration of Guqin [J]. Beauty \& Times, 2005(7): 9-12.

[2] Zhang Yan. On the Relationship between Guqin and Ancient Chinese Literati [J]. Higher Education Forum, 2007(6): 183-185 
[3] Miao Jianhua. A Study on Guqin Aesthetics [M]. Shanghai: Shanghai Conservatory Of Music Press, 2006:60

[4] Huang Xiangpeng. Tradition is a River [A]. On the Inheritance of Ancient Chinese Music — a Study on Music History [C]. People's Music Publishing House, 1992:105-127 